\title{
ENABLING "ENERGY-AWARENESS" IN THE SEMANTIC 3D CITY MODEL OF VIENNA
}

\author{
G. Agugiaro
}

Sustainable Buildings and Cities Unit, Energy Department, AIT - Austrian Institute of Technology, Vienna, Austria giorgio.agugiaro@ait.ac.at

KEY WORDS: Data integration, CityGML, Energy ADE

\begin{abstract}
:
This paper presents and discusses the first results regarding selection, analysis, preparation and eventual integration of a number of energy-related datasets, chosen in order to enrich a CityGML-based semantic 3D city model of Vienna. CityGML is an international standard conceived specifically as information and data model for semantic city models at urban and territorial scale. The still-indevelopment Energy Application Domain Extension (ADE) is a CityGML extension conceived to specifically model, manage and store energy-related features and attributes for buildings.

The work presented in this paper is embedded within the European Marie-Curie ITN project "CINERGY, Smart cities with sustainable energy systems", which aims, among the rest, at developing urban decision making and operational optimisation software tools to minimise non-renewable energy use in cities. Given the scope and scale of the project, it is therefore vital to set up a common, unique and spatio-semantically coherent urban data model to be used as information hub for all applications being developed. This paper reports about the experiences done so far, it describes the test area in Vienna, Austria, and the available data sources, it shows and exemplifies the main data integration issues, the strategies developed to solve them in order to obtain the enriched 3D city model. The first results as well as some comments about their quality and limitations are presented, together with the discussion regarding the next steps and some planned improvements.
\end{abstract}

\section{INTRODUCTION}

Among the possible existing definitions of smart city, it is reasonable to expect that a smart city should create sustainable economic development and high quality of life by excelling in multiple key areas such as economy, mobility, environment, people, living and government. However, excellence can be achieved only by strong integration of human and social capital, as well as ICT infrastructure, which enables, among the rest, to better manage the city's assets, to enhance the quality and performance of urban services, to reduce costs and resource consumption and to improve the contact between citizens and government.

Within the wide spectrum of above mentioned topics and applications, this paper focuses on the energy-related ones, as they play nowadays a very important role in order to achieve the overall goal of a reduction of $\mathrm{CO}_{2}$ emissions. In the smart city context, some of the current research fields deal with adoption of renewables, smart mobility, smart grids, refurbishment, etc. When it comes to buildings, for example, they consume more energy in total than any other sector. An exact knowledge of the current energy demand and the estimation of the future one according to specific scenarios is therefore of high relevance for energy planning at city scale.

Thanks to the constant advances in all fields tied to Geomatics, an increasing number of cities is in the process of creating 3D virtual city models as a means for data integration, harmonisation and storage.

A unique and spatio-semantically coherent urban model can provide a multiplicity of beneficial effects for a smart city, as it represents an information hub for further advanced applications ranging from urban planning, noise mapping, augmented reality, up to energy simulation tools (Bahu et al., 2013; Kaden and Kolbe, 2013; Agugiaro, 2015).
To these extents, CityGML (Gröger and Plümer, 2012) is an international standard conceived specifically as information and data model for semantic city models at urban and territorial scale. It is being adopted and used more and more as integrated and coherent information hub for simulations in different scientific fields, e.g. the energy-related ones.

Although CityGML lacks specific energy-related objects and attributes, it is possible to extend it by means of so-called Application Domain Extensions (ADE). For this reason, a growing international consortium of urban energy simulation developers and users (14 European organisations from 6 countries as of January 2016) has sought since May 2014 to address this need by developing an Energy ADE for CityGML. Some information about the current status of development will be given in the following sections.

The work described in this paper is embedded in the European Marie-Curie ITN project "CINERGY, Smart cities with sustainable energy systems" [CINERGY, 2016], which aims, among the rest, at developing urban decision making and operational optimisation software tools to minimise nonrenewable energy use in cities. The project involves close collaboration between academic research centres and industrial companies from the energy and software technology sector, as well as the municipalities of Geneva and Vienna, which were chosen for their very ambitious sustainability goals.

Given the relevance of the energy themes within the CINERGY project, and starting from a CityGML-based semantic 3D model of Vienna containing mostly only geometries, this paper primarily focuses on describing the tests carried out to understand how to map a number of energy-related datasets available in Vienna to the Energy ADE classes. In addition, as example of an application profiting from the semantically enriched 3D city model, first results are shown when it comes to the estimation of the energy demand for space heating for all residential buildings in the study area. 
The paper is structured as follows: section 2 describes the test area and the data sources, section 3 briefly describes the available $3 \mathrm{D}$ model used in this work, section 4 gives a short overview of the characteristics of the Energy ADE in its current development status, section 5 deals with the data integration issues, the strategies developed to solve them and create the integrated 3D city model. Section 6 presents the first results as well as some comments about their quality and limitations, while section 7 contains the conclusions and the discussion regarding the next steps and some planned improvements.

\section{TEST AREA AND DATA SOURCES}

\subsection{Test area}

Vienna is the capital and largest city of Austria, with a population of nearly 1.8 million. It is composed of 23 districts. The $12^{\text {th }}$ district of Vienna, named Meidling, was chosen as test case. Given its heterogeneity in shape, structure and characteristics, the district of Meidling was deemed adequate to represent a good test case (Figure 1). Meidling spans an area of approximately $8.2 \mathrm{~km}^{2}$, it lies just $5-7 \mathrm{~km}$ southwest of the city centre and is located nearby the well-known Schönbrunn palace. It is a heavily populated urban area (circa 90000 inhabitants, i.e. circa 11000 inhabitants $/ \mathrm{km}^{2}$ ) with many residential buildings of greatly varying size and typology, but also with large recreational areas and parks. It can be approximately divided into two main parts: the north-eastern one is characterised by a heavily developed urban residential texture, while the south-western one is a more mixed (industrial and light residential) area, which then gradually continues southwards to become the $23^{\text {rd }}$ district.

Meidling represents also an important transportation hub, as the homonymous train station lies quite in the middle of the district and serves as an important connecting hub to many bus, tram and rapid transit (S-Bahn and underground) lines between the city and the surrounding suburbs.

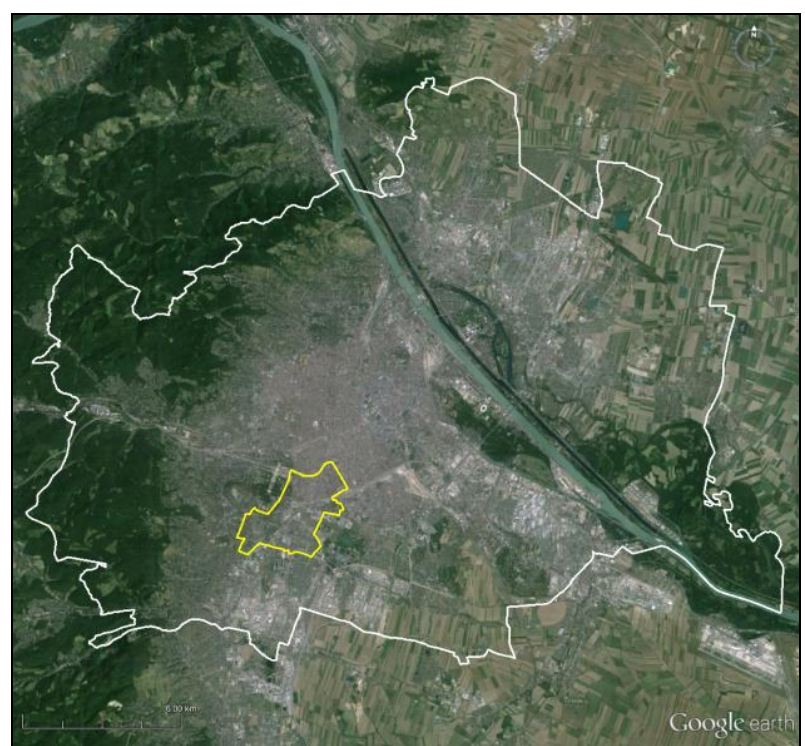

Figure 1. Boundaries of Vienna (in white) and extents of the district of Meidling (in yellow). Image source: Google Earth

\subsection{Spatial data sources}

Several heterogeneous datasets were collected, mainly from the already available Open Government Data Wien catalogue
[OGD-W, 2016], which offers among others WFT and WMS services, or were provided by the Municipality of Vienna for the purposes of the CINERGY project (the latter are identified by an asterisk (*) in the following list). In any case, no new data (spatial and non-spatial) were acquired, the explicit purpose being to use as much existing data as possible, and as much publicly available data as possible.

All spatial data, where applicable, are geo-referenced according to the MGI/Austria GK East projection (EPSG code: 31256), which is the one generally used by the city of Vienna.

In the following, the data sources will be classified into spatial and non-spatial ones, for better reading. Especially in the case of OGD data, they were already retrieved for the whole city.

D1) *A set of CityGML files containing buildings modelled in level of detail LoD0, LoD1 and LoD2. Each file corresponds to a tile of approximately $500 \times 500 \mathrm{~m}$. For Meidling there are 52 tiles covering the whole area of the district. Each CityGML tile is itself the product of a previous data harmonisation and integration process carried out during the summer/autumn of 2015. Some details will be given in section 3 . Following attributes are available at building level (the attribute in italics are stored as CityGML GenericAttributes):

- Building name(s), building address(es),

- Building_class, building_function, building_usage,

- Year of construction, storeys above ground,

- groundArea, lowestRainGutterHeight, ridgeHeigth, lod1 Volume, lod2Volume;

D2) A csv file containing all addresses of Vienna, as well as some ancillary information about building type/use and the coordinates corresponding to each address.

D3) A polygon-based vector map representing the land-use for the whole city of Vienna. Polygons are classified using three hierarchical levels with growing degree of detail;

D4) A set of several point-based vector maps containing information about building names, building use (e.g. schools, theatres, commercial centres, kindergartens, swimming pools, public offices, hospitals, etc.);

D5) Point-based vector datasets with the installed photovoltaic and solar panel installations. The datasets contain information about the installed power and the surface of the solar collectors, and the year of installation;

D6) *A set of raster maps used for the solar cadastre of Vienna, at $0.5 \times 0.5 \mathrm{~m}$ geometric resolution, and consisting in monthly and yearly values of global solar radiation on the roof surfaces, and the corresponding values in terms of potential yield for photovoltaic and solar panel installations (the latter delivered as vector-based maps);

D7) A set of raster maps obtained from the solar cadastre of Vienna and representing 3 classes of roof suitability for installation of solar or photovoltaic panel systems;

D8) A vector-based maps representing the geothermal potential of the underground at different depths;

D9) A raster-based map representing the groundwater thermal potential;

D10) A vector-based map of the wind-potential suitability zones;

\subsection{Non-spatial data sources}

The non-spatial datasets used in this work consist of:

D11) *An Esri Geodatabase-based dataset containing in a number of tables information for each building such as:

- The net floor area classified into different classes (residential, commercial, offices, industry, etc.), - Number of floors above and below ground, 
Year or period of construction

The (unclassified) net floor area for each floor, and the type of floor (cellar, ground floor, attic, etc.),

Address and address ID, to link the records to the spatially defined address dataset (D2)

Further (relatively scarce) information regarding presence of ventilation, heating, domestic hot water preparation systems, etc.

D12) *A Microsoft Excel file containing the Wiener Wärme Kataster (WWK) data - i.e. the "Vienna Heat Cadastre" which contain a number of attributes among which:

Address IDs, e.g. to link the records to the spatially defined address dataset (D2);

Estimated values for annual and monthly energy demand and energy consumption for space heating, domestic hot water and electricity;

D13) An XML-based file containing information about all Gemeindebauten (social housing buildings) in Vienna, such as:

- Address and address ID,

Name of the social housing building,

Year of construction, year of refurbishment,

Number of households,

Name of the architect(s),

Some historical and architectonical information;

D14) *For a limited number of residential buildings (64), a Microsoft Excel file containing information about the number of flats in the building, their floor net area and the precise address with door number for each flat.

It must be stressed that a considerable number of further datasets is already available over the Open Government Data Wien platform. These datasets are surely worth to be integrated into a city model, however they will not be treated in this paper as they are - for now - out of scope.

\section{THE 3D CITY MODEL}

The CityGML-based semantic city model of Meidling is itself the product of a data harmonisation and integration process and acts as a prerequisite for the work described in this paper.

Originally, a set of CityGML files containing buildings modelled only in LoD2 was delivered by the city of Vienna within the framework of the CINERGY project. The dataset is not currently publicly available yet, as internal data quality and consistency checks are still being carried out prior to the official publication. Nevertheless, the whole dataset (1460 tiles) was used in this work for testing purposes, in order to explore and test the data, and prepare in advance the required data integration procedures. Once the quality tests will be complete, the "official" CityGML dataset will be used instead of the current one.

Giving a detailed description of the $3 \mathrm{D}$ model is beyond the scope of this paper, however further details can be found in Agugiaro (2016). In the following, only the main characteristics will be mentioned.

The 3D model of Meidling contains buildings modelled in LoD0, LoD1 and LoD2. Buildings are modelled either as multipart objects, i.e. as a composition of CityGML BuildingParts, or as simple single-part objects. For some buildings, architectonical elements like "flying roofs" are added as Building Installations in LoD2.

Examples of possible problematic cases that may arise when automatically generating single or multi-part buildings from the input datasets are presented in Figure 2. Case a) is a correct building, cases b) and c) represent groups of buildings that should be separated, while case d) is a building modelled as a single geometrical object, although in reality it generally corresponds to a complex "agglomerate", made up of several parts.

For the cases b) and c) a hierarchical reclassification of the input geometries had to be carried out, while for case d) the only solution would be to split the geometries (e.g. with a sort of vertical cut like and ideal cookie cutter), and then classify them again. It was however decided not to solve case d) as this operation would break the consistency with the original data from the Municipality of Vienna.

All 52 CityGML tiles were reprocessed: the reclassification and regrouping led to 7390 CityGML buildings, of which $39.6 \%$ are single-part buildings and the remaining are multi-part buildings.
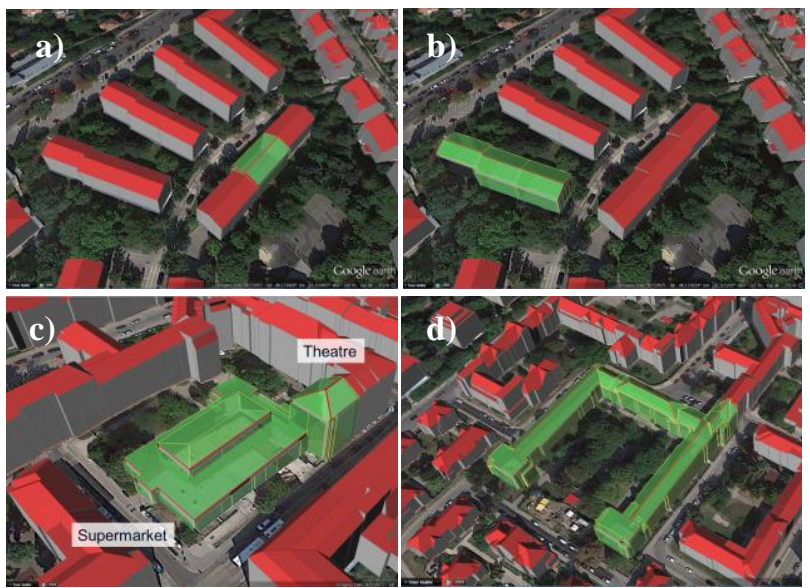

Figure 2. In green: a) a correctly modelled building; b) 3 similar residential buildings wrongly grouped into a single building; c) 2 different buildings wrongly grouped into a single building; d) a building "agglomerate" derived from one single footprint

\section{THE ENERGY ADE}

When dealing with urban energy modelling, a number of open model standard exists, the common goal being to ease interoperability between heterogeneous software platforms. However, they are strongly dependent on the scale and application domain they are conceived for.

For single buildings - it is the case of the Building Information Model (BIM) domain - the two most known and commonly adopted open standards are either IFC (Industry Foundation Classes) or gbXML (green building XML). The former is intended to describe building and construction-industry data. The latter acts similarly and is as an open schema for sharing building information between disparate building design and simulation software tools. Both data models cover both 3D geometry and semantics.

When working at city scale, however, the number of choices with regards to open and standard data models comprises essentially only CityGML and (at least for Europe) the upcoming INSPIRE Data Specification on Buildings (which, in part, takes inspiration from CityGML itself).

What is more, when it comes to urban energy data models, even CityGML falls short. CityGML can already store attributes with regards to the year of construction, the building class and usage, however not all energy-related attributes and features can be stored natively in a systematic and standard way.

To add to the complexity, also the variety of methodologies for urban energy modelling can vary greatly. A detailed overview is given in Swan and Ugursal (2009), according to whom two main approaches can be identified: a top-down one, which is 
based generally on aggregated econometric and technological city-wide data, and a bottom-up one, which uses detailed statistical data (e.g. based on historical energy consumption data) coupled with engineering data (e.g. based on physical models). What is more, the existing tools for assessment of urban energy-topics (e.g. CitySim [CITYSIM, 2016]) very often rely on proprietary data formats, however a growing number of new tools are adopting CityGML as common data model (e.g Simstadt [SIMSTADT, 2016], Teaser [TEASER, 2016]), given the possibility to extend it by means of so-called Application Domain Extensions (ADE): depending on the specific needs, new features or properties can be simply added, hence augmenting its modelling capabilities.

For this reason, an international consortium of urban energy simulation developers and users has started in May 2014 to develop an Energy ADE for CityGML.

The CityGML Energy Application Domain Extension (ADE) is conceived to extend CityGML and allow a standardised way of representing, storing and exchanging energy-related features and attributes which are of relevance for urban energy models. It is intended to deal with heterogeneous data qualities, levels of details and urban energy models complexities (e.g. from monthly energy balance of ISO 13790 , to sub-hourly dynamic simulations). It takes into consideration the INSPIRE Directive of the European Parliament, as well as the recent US Building Energy Data Exchange Specification (BEDES) (Nouvel et al., 2015).

The Energy ADE focuses on the building, its physical properties and the systems installed in it. It is not meant to cover urban centralized energy infrastructures, like district heating system or gas network, as they are already represented by the CityGML Utility Network ADE (Becker et al., 2013). Nevertheless, the Energy ADE allows for interfacing with the utility networks through substation node objects.

The Energy ADE is structured in a modular way. For space reasons, only a limited number of classes relevant to the work presented in this paper will be briefly presented. Further information, as well as the Energy ADE specifications are freely accessible on the [SIG3D-EADE, 2016] and [ENERGY-ADE, 2016] websites, where detailed explanations and examples are given. In the core module (Figure 3) the standard CityGML _AbstractBuilding is extended with a number of attributes like attic and basement type (e.g. conditioned or not), the building type (e.g. single family house, apartment block, etc.), the construction style, different types of floor area, refurbishment measures, as well as the energy performance certification.

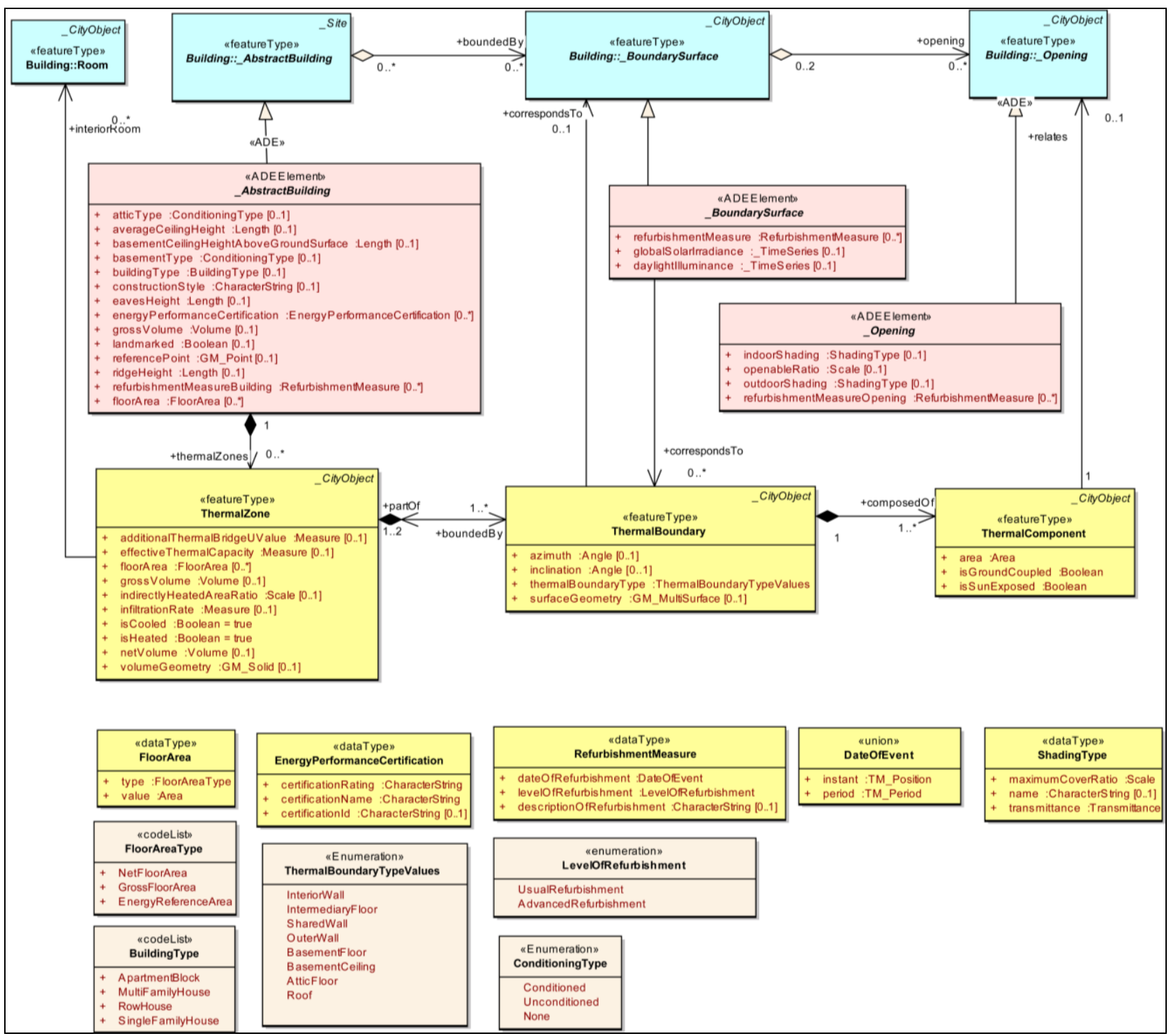

Figure 3. UML diagram of the core module in the Energy ADE v. 0.6. Image source: courtesy of Energy ADE workgroup 
Analogously, the _BoundarySurface and _Opening classes are extended with other typical energy-relevant attributes (e.g. global solar irradiance, etc.). A building may have one or more ThermalZone objects, which are defined by a number of ThermalBoundary objects. These can be either semantically defined and linked to CityGML_BoundarySurfaces, or explicity modelled geometrically by means of multisurfaces.

The occupancy module (Figure 4) characterises the buildings in terms of UsageZone objects, i.e. zones with a homogeneous usage (e.g. residential) in terms of, for example, common heating, ventilation and cooling schedules. A UsageZone can be associated to a ThermalZone and contains one (or multiple) BuildingUnit objects, i.e a dwelling or a workplace. This object is defined by the owner's information as well as the number of rooms and the floor area. Both BuildingUnit and UsageZone may have one or several Occupants and Facilities objects. The object Occupants represents a homogeneous group of occupants.

The energy and systems module (not depicted) contains information concerning the energy forms (energy demand, energy source) and the energy systems (conversion, distribution and storage systems). In particular, the EnergyDemand object can be associated to any _CityObject (e.g. _AbstractBuilding, ThermalZone, UsageZone, BuildingUnit, etc.) and represents therefore the connection of the module to the rest of the Energy ADE. One further module, the constructions and materials module (not depicted) contains the physical characterization of building construction elements and can be associated with any CityGML_CityObject. It is applicable to simply store U- and gvalues for walls and windows, but also to model more complex, multi-layered construction elements.

All modules depend on a common module, the time series and schedules module (not depicted), in which time-dependent variables (regular and irregular time series) and different types of schedules are defined as data types to be used by all Energy ADE elements.

The inclusion of temporal variables, and the definition of their relevant properties such as the acquisition method (e.g. simulation or metering) allow to model and store both simulation results and metering data. Moreover, different time steps, corresponding to different building simulation, methods and metering systems can be used.

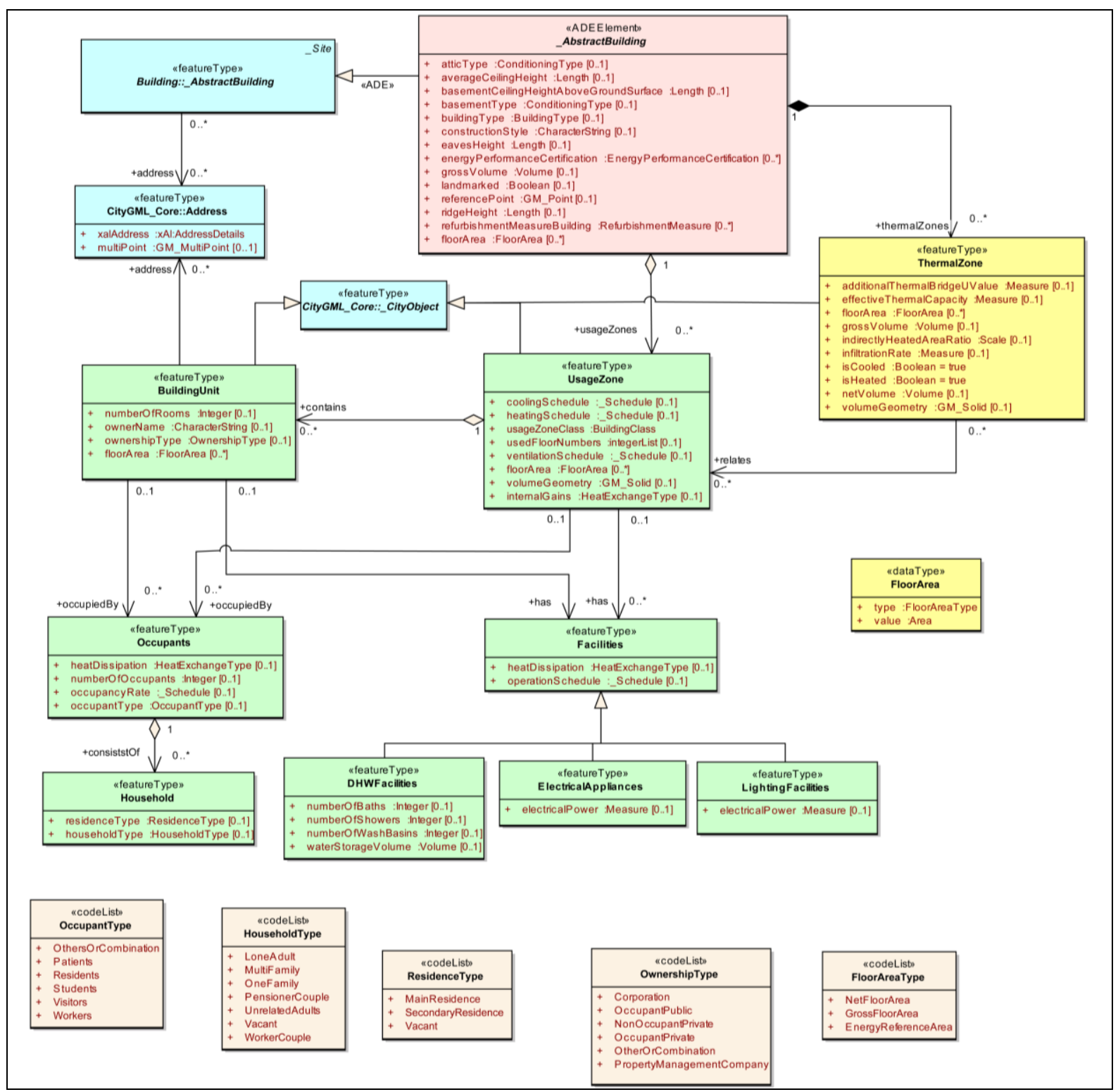

Figure 4. UML diagram of the occupancy module in the Energy ADE v. 0.6. Image source: courtesy of Energy ADE workgroup 


\section{DATA INTEGRATION AND "ENRICHMENT" OF THE 3D CITY MODEL}

All spatial and non-spatial datasets briefly described in section 2 were explored to identify potential issues with regards to their integration. One goal was to enrich the geometries (in LoD0, LoD1 and LoD2) by means of attributes from the existing datasets in order to create a unique data source as coherent and "clean" as possible.

One further goal was to test how and to which extent the available datasets can be mapped to the Energy ADE, or whether and how the Energy ADE can be further enhanced.

In order to facilitate data exploration and analysis, all datasets were first imported into a PostgreSQL 9.4 database with the PostGIS 2.2 extension. All 52 CityGML tiles were imported into the already predefined citydb schema using the freely available 3DcityDB tools. The UML diagram of the Energy $\mathrm{ADE}$ was used as reference to design and add the database entities and relations needed for the Energy ADE.

Once the mapping between the input datasets and the destination CityGML/Energy ADE entities was completed, the data integration process was carried out by means of a series of workbenches in Safe Software's FME 2015 Professional or as SQL scripts. Not all available datasets and their integration will be described in detail, but only those where relevant issues were identified and solved. Although applied only to the study area in Meidling, all integration strategies were conceived to be replicable in any part of the city.

First of all, due to the publication as Open Government Data of the official dataset with all addresses for Vienna (dataset D2 in section 2.1) by December 2015, and due to the delivery from the city of Vienna of the detailed data for each building (D11) shortly before, it was decided to replace most of the attributes already integrated in the CityGML tiles with the content of the new datasets, which were deemed more reliable and updated.

Nevertheless, the original GenericAttributes lod2Volume, lowestEavesHeight and ridgeHeight were mapped to the corresponding ones in the Energy ADE building class.

Given the lack of any common key between the addresses and the building geometries, the only integration strategy relied on simple spatial overlay. For this reason, a check was carried out to identify whether and how many points really fall within the footprints of the buildings. Thanks to this check, which was one of the most time-demanding in the whole pipeline, the wrong point geometries for Meidling were edited and - if necessary moved manually, as no unique, satisfying and reliable automatic criterion could be found to assign a point to the corresponding building geometry. An example can be seen in Figure 5.

Once these checks and edits were finished, for the study area of Meidling the number of points correctly falling inside a building polygon rose from $88.9 \%$ to $95.4 \%$, out of 7456 total points. For some addresses it was possible to extract information about the name of the building or its usage (museum, hospital, etc.) and to assign it to the CityGML building_usage attribute.

As a consequence, the geo-referenced dataset containing all addresses was used also in successive steps, as it represents the actual link between the building geometries and other nonspatial datasets, whenever no other data integration technique is possible but spatial overlay. For example, when it comes to the buildings dataset (D11), the year of construction, the number of storeys above and below ground were mapped to standard CityGML attributes. For each building, and for each class of net floor area in that building, a UsageZone object was created and the corresponding value of net floor area stored. The area classification (residential, office, etc.) was also mapped to the

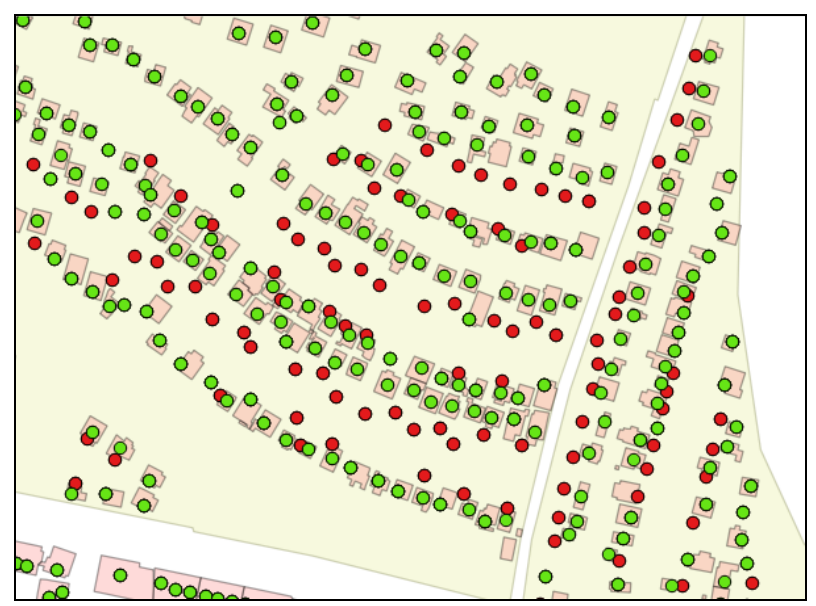

Figure 5. Example of addresses falling outside the building geometries (in red) and after editing (in green)

building_function attribute in CityGML. From the connected dataset containing the (unclassified) net floor area for each floor, the floor classification information was used to defined whether a building has a conditioned attic or not, thus mapping this information to the Energy ADE atticType attribute for the buildings.

With regards to the WWK dataset (D12), the yearly and monthly values of energy demand and consumption for heating, cooling and electricity where stored as EnergyDemand objects using the datatype for time series provided by the Energy ADE. With regards to the social housing dataset (D13), the information about the type and date/period of refurbishment could be stored in the corresponding entities of the Energy ADE. The name, the building usage, the date of construction were mapped to standard CityGML attributes.

For dataset D14, the detailed information about net floor area and precise address of each flat where mapped to Energy ADE BuildingUnit objects, in relation to the corresponding UsazeZone classified as "residential".

In case of the spatial data, dataset D3 representing the land-use for the whole city of Vienna was imported as CityGML LandUse objects. From dataset D4 information was extracted and mapped to standard CityGML building_name and building_usage attributes. For dataset D5, the data about the installed photovoltaic and solar thermal systems were mapped to PhotovoltaicSystem and SolarThermalSystem objects (energy systems module) in the Energy ADE.

From the rasters in dataset D6, the monthly and yearly values of global solar irradiance on each roof surface where computed and mapped to the Energy ADE _BoundarySurface attribute globalSolarIrradiance as time series data.

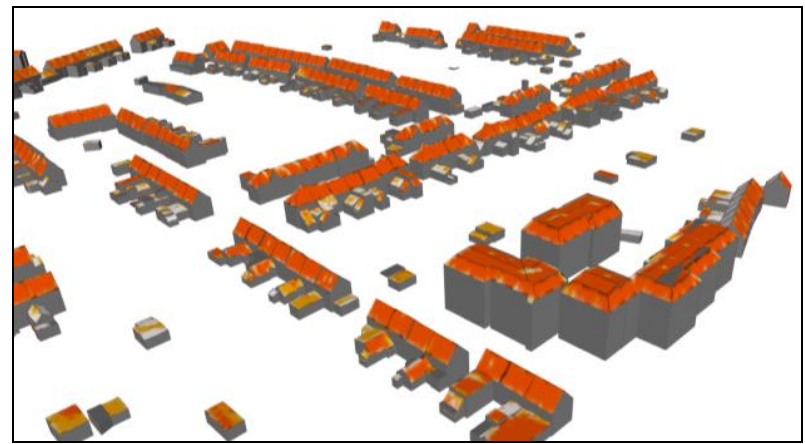

Figure 6. Texturization of the building roofs to show the suitability for installation of PV and solar thermal systems 
The raster maps in dataset D7 were instead used for visualisation purposes in that they were applied as textures to the roof surfaces. An example is shown in Figure 6.

The remaining datasets (e.g. D8, D9 and D10) have not been integrated in CityGML (yet), but nevertheless they were harmonised and stored in the same PostgreSQL database. The reason for this is that values of potentials (geothermal, windpotential, etc.) are not currently modelled in the Energy ADE although they can indeed be already stored natively simply as GenericObjects or GenericAttributes in CityGML. Nevertheless, this is one of the points that will be discussed for future Energy ADE releases.

\section{RESULTS}

At the end of the integration process, nearly all of the available datasets listed in section 2 could be integrated into the $3 \mathrm{D}$ city model. The Energy ADE, in its version 0.6 at the time of writing (January 2016), made it possible to store nearly all feature and attributes that were deemed meaningful to be added to the city model.

When it comes to completeness of the attributes, some initial tests and visual inspections were carried out to check the overall results of the data integration process. In the following, some attributes are discussed. Percentages are referred to the total number of buildings in the Meidling city model (7390).

Regarding the building function, completeness accounts for about $81.3 \%$ of all buildings, however some inconsistencies were found. For example, it was observed that a church is assigned only residential net floor area, or that in a school there is net floor area for offices, but no net floor area classified for education. For the building usage, the information contained is indeed detailed and no relevant errors were found, however it is quite scarce as it accounts for only $5.3 \%$ of all buildings. One reason for this is that information about private residential buildings is not included in the original input datasets, unlike other classes like schools, cinemas, offices, etc.

With regards to the year of construction, about $81.4 \%$ of all buildings have this information. For the number of storeys above ground, completeness accounts for about $81.3 \%$ of all buildings $(78.8 \%$ for number of storeys below ground), however some errors were found by means of simple visual inspection and comparison with Google Maps (oblique views). For this reason, a check routine is being implemented which confronts the given value with a computed one. This will help identify and possibly correct gross discrepancies.

Finally, a comparison was carried out between the total net floor area of 64 buildings, for which data are available both from dataset D11 and D14. On average, the D14 net floor areas values are $2.1 \%$ bigger than the D11 ones, however with significant discrepancies especially with smaller buildings. An excerpt with only 9 buildings is presented in Table 8 .

A number of other checks is in preparation to identify other inconsistencies and help correct them.

In order to test and exploit the added value of a harmonised and unique data source, an application was developed to estimate the energy demand for space heating of all residential buildings in the test area in order to obtain a city-wide energy mapping of the (residential) built environment.

For example, thanks to the availability of the LoD2 geometries, all shared walls (between adjacent BuildingParts and between adjacent buildings) could be computed and classified. The availability of the Energy ADE allowed to compute the geometries of the ThermalZone once and to store them for future retrieval and further use (Figure 9, top).
The energy demand was computed on the basis of the guidelines issued by the Austrian Institute of Construction Engineering and starting from the semantic 3D city model which acted as information hub containing the required spatial and non-spatial data (Figure 9, bottom). All relevant implementation steps, the first results, the planned enhancements and the future steps are described in details in Agugiaro and Möller (2016).

\begin{tabular}{|l|r|r|r|}
\hline Bdg_id & $\begin{array}{c}\text { D11 } \\
{\left[\mathbf{m}^{\mathbf{2}}\right]}\end{array}$ & $\begin{array}{c}\text { D14 } \\
{\left[\mathbf{m}^{\mathbf{2}}\right]}\end{array}$ & \multicolumn{1}{c|}{$\begin{array}{c}\text { Diff } \\
{[\mathbf{\%}]}\end{array}$} \\
\hline 55859 & 335 & 260 & -22.5 \\
\hline 55861 & 195 & 260 & 33.5 \\
\hline 55881 & 3389 & 3564 & 5.2 \\
\hline 55979 & 1482 & 1433 & -3.3 \\
\hline 56052 & 2025 & 2098 & 3.6 \\
\hline 56059 & 537 & 575 & 7.1 \\
\hline 56060 & 590 & 576 & -2.4 \\
\hline
\end{tabular}

Table 8 Example of total net floor area for each building according to datasets D11 and D14 and their difference

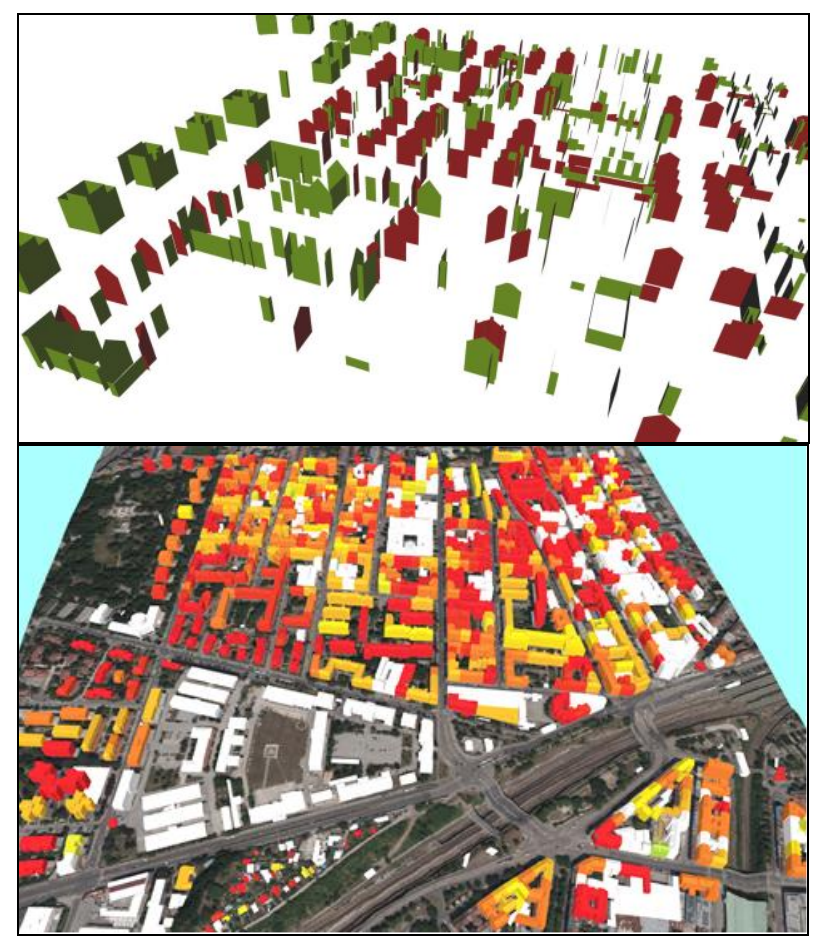

Figure 9. Top: Shared walls between adjacent buildings (in red) and between adjacent BuildingParts (in green). Bottom: Annual energy demand for space heating of residential buildings.

Colours correspond to energy classes as in the guidelines issued by the Austrian Institute of Construction Engineering

\section{CONCLUSIONS AND OUTLOOK}

Within the wide spectrum of smart cities topics, this paper has dealt specifically with the adoption of an open and standard data model for semantic 3D virtual city models (CityGML), as well as its extension by means of an Energy Application Domain Extension (ADE), in order to enhance interoperability in the context of energy applications. A district in the city of Vienna was used as test case study.

In particular, this paper has dealt with two specific aspects: 1) information fusion of heterogeneous data sources into one consistent semantic 3D city model, and 2) discussion of concrete data integration issues which can be used as best 
practice and an indicator for the efforts that other cities would have to reckon with for a similar approach.

The integrated, semantic, 3D, virtual, and CityGML-based city model of the city of Vienna is now "energy-aware", in that it integrates also energy-relevant features and attributes.

The heterogeneity of input datasets has proven the versatility of the Energy ADE to accommodate a valuable number of energyrelated data. Although, in general, data have been gathered for the whole city, the district of Meidling was chosen as study area: tests were carried out in order to acquire knowledge about the data, gather experience how to prepare and (re)structure them in order to facilitate the data integration process.

The main data integration issues (semantic and structural problems, cardinality and geometry issues, etc.) were identified, a set of rules was set to overcome most of these issues. A stepbased workflow was implemented that allows to obtain a semantic 3D model based on the CityGML standard and adopting the Energy Application Domain Extension to store specifically energy-related features and attributes.

Therefore, the results obtained and the experience gathered so far are satisfactory and will be further exploited when extending the approach to the whole city.

The Energy ADE, in its version 0.6, has shown no significant drawbacks, nevertheless further improvement can be achieved only by means of testing with real-life situations. To this extent, the case study of Meidling has contributed positively to the further development process of the Energy ADE. Some of the aspects that need to be further tackled are for example how to coherently store information about buildings protection levels (e.g. cultural heritage buildings), as this might reduce/influence the refurbishment alternatives, or how (and whether) to store information about energy potentials.

In order to test and exploit the added value of the harmonised and unique data source, an application was developed which estimates the energy demand for space heating of all residential buildings in the study area, in that it retrieves nearly all required input data from the city model, and it further benefits of it by storing back results as well as further intermediate processing results (e.g. shared walls), thus eliminating the need to compute them again every time a simulation is run.

Nevertheless, the application mentioned in this paper is to be intended only as an example, as the 3D city model could to be used in a number of other applications, given its open standard and its deliberately application-independent nature.

In the near future, the "energy-aware" city model will be coupled to further energy-related software tools (e.g. EnergyPlus) (Agugiaro et al., 2015), in order to exploit the functionalities of full-fledged dynamic simulation tools and to further refine the simulation results. At the same time, different energy scenarios could be defined and their effect on existing infrastructures (e.g. district heating) estimated.

\section{ACKNOWLEDGEMENTS}

The author gratefully acknowledges the European Commission for providing financial support during the conduct of research under the FP7-PEOPLE-2013 Marie Curie Initial Training Network "CINERGY" project with Grant Agreement Number 606851. As stated and indicated in section 2.2, some of the datasets used in this work were kindly provided by the city of Vienna specifically for CINERGY project. The author would like to thank in particular Harald Reichl (Magistratsdirektion Strategische Energieangelegenheiten), Hubert Lehner and Christian Sam (MA 41 Stadtvermessung) and Christian Pöhn (MA 39 Prüf-, Überwachungs- und Zertifizierungsstelle).
Finally, the author is grateful to Britta Kunert and Christoph Nachtigal for their precious help during the preparation of the input datasets.

\section{REFERENCES}

Agugiaro, G., 2015, Energy planning tools and CityGML-based $3 \mathrm{D}$ virtual city models. Experiences from Trento (Italy). Applied Geomatics, 8(1), pp. 41-56.

Agugiaro, G., 2016, First steps towards an integrated CityGMLbased 3D model of Vienna, Annals of the Photogrammetry, Remote Sensing and Spatial Information Sciences, III-4, pp. 139-146

Agugiaro, G., Hauer, S., Nadler, F., 2015, Coupling of CityGML-based semantic city models with energy simulation tools: some experiences. REAL CORP 2015 Proceedings/Tagungsband, Gent, 5-7 May 2015, pp. 191-200.

Agugiaro, G., Möller, S., 2016, Gebäudescharfe Schätzung des Heizwärmebedarfs anhand des semantischen 3D-Stadtmodells Wiens. AGIT - Journal für Angewandte Geoinformatik, 2-2016. Herbert Wichmann Verlag, pp. 124-133.

Bahu, J.M., Koch, A., Kremers, E., Murshed, S.M., 2013, Towards a 3D spatial urban energy modelling approach. Annals of the Photogrammetry, Remote Sensing and Spatial Information Sciences, II-2/W1, pp. 33-41.

Becker, T-, Nagel, C., Kolbe, T.H. (2013) Semantic 3D modeling of multi-utility networks in cities for analysis and 3D visualization. Progress and New Trends in 3D Geoinformation Sciences. Springer Verlag Berlin, Heidelberg, pp. 41-62.

Gröger, G., Plümer, L., 2012, CityGML - Interoperable semantic 3D city models. ISPRS Journal of Photogrammetry and Remote Sensing, 71, pp. 12-33.

Kaden, R., Kolbe, T.H., 2013, City-wide total energy demand estimation of buildings using semantic 3D city models and statistical data. Annals of the Photogrammetry, Remote Sensing and Spatial Information Sciences, II-2/W1; pp. 163-171.

Nouvel, R., Bahu, J.M., Kaden, R., Kaempf, J., Cipriano, P., Lauster, M., Haefele, K-H., Munoz, E., Tournaire, O., Casper, E., 2015b, Development of the CityGML Application Domain Extension Energy for Urban Energy Simulation. Proc. of 4th International Conference of the International Building Performance Simulation Association (IBPSA).

Swan, L.G., Ugursal, V.I., 2009, Modeling of enduse energy consumption in the residential sector: A review of modeling techniques. Renewable and Sustainable Energy Reviews, pp. $1819-1835$.

\section{REFERENCES FROM WEBSITES (31 MAY 2016)}

CINERGY, http://www.ci-nergy.eu CITYSIM, http://citysim.epfl.ch/ ENERGY-ADE, http://en.wiki.energy.sig3d.org OGD-W, https://open.wien.gv.at SIG3D-EADE, http://www.sig3d.org/citygml/2.0/energy/ SIMSTADT, http://www.simstadt.eu TEASER, https://github.com/RWTH-EBC/TEASER 